Pädiatrie up2date

$4 \cdot 2019$

\title{
Chirurgie/Orthopådie 10
}

\section{Unfall oder Kindesmisshandlung? Differenzialdiagnostik bei Frakturen ohne klare Ursache}

Ramona Ajiri Friederike Koerber Sibylle Banaschak

Oliver Semler 


\section{Unter dieser Rubrik sind bereits erschienen:}

Update Hüftreifungsstörung - Diagnostik und Behandlung T. Baumann, R. Schmid, S. Essig Heft 4/2017

Schädeldeformitäten - Kraniosynostosen und lagebedingte Wachstumsstörungen J. E. Zöller, A. Grandoch Heft 4/2017

Kinderorthopädie - Schwerpunkt Fuß M. Wachowsky,

O. Eberhardt, T. Wirth Heft 2/2016

Erkrankungen der Vorhaut T. Klein, R. Vahdad, T. Boemers Heft 3/2015

Ösophagusatresie S. Loff, U. Mehlig Heft 2/2015

Sportorthopädische Probleme im Kindes- und Jugendalter O. Eberhardt, T. Wirth Heft 4/2011

Achs- und Rotationsdeformitäten P. Schmid Heft 3/2010

"Das gerade Kind" - kindliche Wirbelsäule und Skoliose

K. Kafchitsas, T. Vetter, A. Kurth Heft 2/2010
Genitale Fehlbildungen und Abweichungen der geschlechtlichen Differenzierung B. Detlefsen, E. Korsch, T. Boemers Heft 4/2009

Lippen-Kiefer-Gaumen-Segelspalten R. Sader Heft 2/2009

Minimalinvasive Kinderchirurgie H. Till, U. Rolle Heft 1/2009

Frakturen P. Schmittenbecher Heft 1/2008

Kleine kinderchirurgische Notfälle G. Baumgartner Heft $1 / 2008$

Präoperative Vorbereitung J. Strauß, K. Becke Heft 4/2007

Hüftgelenksdysplasie R. Graf Heft 3/2007

Hodenhochstand H. Lochbühler Heft 2/2006

\section{ALLES ONLINE LESEN}

Mit der eRef lesen Sie Ihre Zeitschrift: online wie offline, am PC und mobil, alle bereits erschienenen Artikel. Für Abonnenten kostenlos! https://eref.thieme.de/paed-u2d

\section{JETZT FREISCHALTEN}

Sie haben Ihre Zeitschrift noch nicht freigeschaltet? Ein Klick genügt: www.thieme.de/eref-registrierung 


\title{
Unfall oder Kindesmisshandlung? Differenzialdiagnostik bei Frakturen ohne klare Ursache
}

Ramona Ajiri, Friederike Koerber, Sibylle Banaschak, Oliver Semler

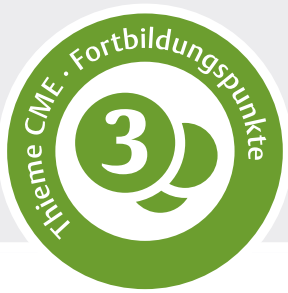

\begin{abstract}
Frakturen im Kindesalter können nicht nur Folge eines Unfalls, sondern auch einer Kindesmisshandlung sein. Diese Differenzierung ist gerade im Kleinkindalter häufig schwierig, für das weitere Vorgehen und die Zukunft der Patientin oder des Patienten aber von größter Bedeutung. Nach Anamneseerhebung und klinischer Untersuchung dienen Röntgenaufnahmen, Laborwerte und weitere Untersuchungen zur Frakturdiagnose und zum Ausschluss anderer Erkrankungen.
\end{abstract}

Der Verdacht auf eine Kindesmisshandlung entsteht häufig, wenn die Anamnese für den vorliegenden Befund nicht plausibel erscheint. Diagnostisch ist das RöntgenSkelettscreening Mittel der Wahl. Eine Knochenszintigrafie oder ein Babygramm werden nicht mehr durchgeführt. Multiple Frakturen in verschiedenen Heilungsstadien, metaphysäre Frakturen der langen Röhrenknochen und Rippenserienfrakturen machen eine Misshandlung als Ursache wahrscheinlich, wohingegen Wirbelkörperfrakturen eher auf eine systemische Skeletterkrankung hindeuten. Beim Verdacht auf eine Kindesmisshandlung sollte die behandelnde Ärztin bzw. der behandelnde Arzt ein multiprofessionelles Vorgehen einleiten und lokale Strukturen (Kinderschutzgruppe, Kinderschutzambulanz) nutzen.

\section{Einleitung}

Frakturen im Kindesalter stellen die behandelnde Ärztin bzw. den behandelnden Arzt oft vor Herausforderungen. Die direkte Diagnose mittels Röntgen und die konservative oder operative Versorgung sind dabei nicht so schwierig wie die Klärung des Entstehungsmechanismus. Dieser ist aber entscheidend, um eine Kindesmisshandlung und damit eine weitere Gefährdung des Patienten nicht zu übersehen.

Eine Kindesmisshandlung ist eine durch aktives Handeln oder durch Unterlassen von entsprechenden Maßnahmen eintretende Schädigung von Körper und Psyche eines Kindes. Das Spektrum möglicher Misshandlungsformen ist breit und reicht von körperlicher Misshandlung (Schläge mit der Hand, mit Gegenständen etc.) über alle Formen von Vernachlässigung (körperlich, seelisch/emotional) bis zum sexuellen Missbrauch. Oft kommt es bei einer körperlichen Misshandlung auch zu Schädigungen am muskuloskelettalen System des Kindes in Form von Frakturen.

\begin{abstract}
Merke
Eine Kindesmisshandlung kann sowohl durch aktives Handeln als auch durch Unterlassen entsprechender Maßnahmen eintreten.

In diesem Beitrag soll es um die Diagnostik und Differenzialdiagnose von Frakturen gehen, bei denen auch eine Kindesmisshandlung als Ursache möglich erscheint. Im vorliegenden Kontext können mehrere Formen von Misshandlung in Betracht kommen: die aktive Verletzung des Kindes, die Vernachlässigung der adäquaten Aufsicht mit der Folge von Unfällen und bei chronisch vorerkrankten Kindern eine Schädigung durch einen inadäquaten Umgang mit einer bekannten Erkrankung.
\end{abstract}

Internationalen Studien zufolge wurden ca. 25\% aller Erwachsenen Opfer einer Kindeswohlgefährdung in ihrer eigenen Kindheit. Dem 2018 veröffentlichten europäischen Sachstandsbericht der Weltgesundheitsorganisation zufolge besteht noch erheblicher Handlungsbedarf zur Prävention von Kindesmisshandlung [1]. Die von einer Kindesmisshandlung betroffenen Personen weisen häufig kurzfristige und langfristige physische sowie psychische Folgen auf. Kindesmisshandlung ist ein gesellschaftliches Problem mit verheerenden Folgen, unter denen die Betroffenen oft bis ins Erwachsenenalter leiden.

Frakturen sind nach Weichteilverletzungen der zweithäufigste Befund bei einer körperlichen Kindesmisshandlung. Diese stellen eine besonders gewalttätige Form der Kindesmisshandlung dar, da erhebliche Kräfte benötigt werden, bis ein Knochen bricht. Insbesondere Kinder unter 3 Jahren sind von misshandlungsbedingten Frakturen betroffen, wohingegen akzidentelle Frakturen eher bei älteren Kindern vorkommen. Bei der Beurteilung von Frakturen gilt generell: Je immobiler das Kind, desto wahrscheinlicher ist es, dass eine Fraktur nicht akzidentell verursacht wurde. Das Erkennen und Verhindern einer 
Kindesmisshandlung ist wichtiger Bestandteil der ärztlichen Aufgabe und erfordert eine interdisziplinäre Herangehensweise. Übersehene Misshandlungen weisen eine hohe Rezidivrate auf und gefährden den weiteren Lebensweg der Betroffenen.

Das ärztliche Vorgehen sollte immer am Kindeswohl orientiert sein. Bei Verdacht auf eine Kindeswohlgefährdung empfiehlt sich die Einbeziehung einer Kinderschutzgruppe bzw. Kinderschutzambulanz (je nach lokaler Struktur), um interdisziplinär über diagnostische Maßnahmen und das weitere Vorgehen (diagnostische Maßnahmen, Einschaltung des Jugendamtes etc.) zu beraten. Eine Kinderschutzgruppe in der Nähe lässt sich z. B. auf der Homepage der Deutschen Gesellschaft für Kinderschutz in der Medizin (www.dgkim.de) finden oder kann über die jeweilige Ärztekammer erfragt werden.

Im Rahmen gerichtlicher Verfahren (Straf- oder Familienrecht) wird gelegentlich der Ausschluss einer Knochenerkrankung gefordert. Dies ist aus wissenschaftlicher Sicht eine unmöglich zu erfüllende Forderung. Mithilfe geeigneter Untersuchungen können aber häufige Ursachen von Frakturen bestätigt oder ausgeschlossen werden. So können klinisch relevante Erkrankungen, die zu einer Instabilität des Skelettsystems führen, untersucht und auch ausgeschlossen werden.

Zwei weitere Aspekte müssen in diesem Zusammenhang kurz angesprochen werden: Die Erfindung der „temporary brittle bones“ ist hinreichend widerlegt [2]. Auch die Frage, ob eine Rachitis vorliegt, wird oft diskutiert. Abgesehen davon, dass eine Rachitis, sollte sie wirklich in so schwerer Form vorliegen, eine massive medizinische Vernachlässigung belegt, sollte auch bei angeblichen Auffälligkeiten bei Stoffwechselmesswerten zu der klinischen Relevanz oder Nichtrelevanz in der Gesamtbeurteilung Stellung genommen werden. Eine isolierte Betrachtung von Messwerten ohne Bewertung der klinischen Situation des Kindes ist unwissenschaftlich.

\section{Klassifikation von Frakturen im Kindesalter}

Frakturen können auf unterschiedliche Arten klassifiziert werden. Eine mögliche Einteilung folgt der Lokalisation der Frakturen. So können Frakturen an Extremitäten von solchen an den Wirbelkörpern oder am Schädel unterschieden werden. An den Extremitäten ist eine weitere Aufschlüsselung in meta-, epi- oder diaphysäre Frakturen möglich und liefert unterschiedliche Hinweise auf den Entstehungsmechanismus der Fraktur.

Des Weiteren werden Frakturen danach eingeteilt, ob es sich um traumatische (akzidentelle) Frakturen oder um pathologische Frakturen (ohne adäquates Trauma) han-

\section{ÜBERBLICK}

Systemische Erkrankungen, die mit einer erhöhten Frakturgefahr verbunden sein können

\section{Endokrine Störungen}

- Thyreotoxikose

- Diabetes mellitus

- Hypogonadismus

- Cushing-Syndrom

\section{Gastrointestinale Störungen}

- Malabsorption

- Hepatitis

- Gallengangatresie

\section{Stoffwechselerkrankungen}

- Glykogenose

- Homozystinurie

- lysinurische Proteinintoleranz

- Tyrosinämie

- Kollagensynthesestörungen (Osteogenesis imperfecta, Marfan-Syndrom, Ehlers-Danlos-Syndrom)

\section{Andere Ursachen \\ - Leukämien \\ - Anorexia nervosa \\ - zyanotische Herzfehler \\ - Medikamente: Glukokortikoide, Antikonvulsiva \\ - Immobilisationsosteoporose}

delt. Hierbei ist zu beachten, dass es keine allgemein anerkannte Definition von einem „adäquaten Trauma“ gibt, sondern dass jeweils geprüft werden muss, ob die anamnestisch angegebene Ursache mit dem Verletzungsmuster übereinstimmt. Es ist besonders wichtig, die Art der Krafteinwirkung zu erfassen, da die Röhrenknochen bei axialen Krafteinwirkungen wesentlich stabiler sind, als wenn eine ähnliche Kraft im Sinne eines Rotationstraumas auf den Knochen ausgeübt wird.

Neben der Klassifizierung nach Ort und einwirkender Kraft spielt auch eine mögliche Grunderkrankung eine Rolle, um Frakturen zu beurteilen. Es müssen angeborene Skelettdysplasien und Mineralisierungsstörungen ebenso beachtet werden, wie z. B. der Einfluss von Medikamenten und anderen Erkrankungen, die das muskuloskelettale System beeinflussen.

Eine Kindesmisshandlung sollte in die differenzialdiagnostischen Überlegungen einbezogen werden, wenn der von den Betreuungspersonen angegebene Entstehungsmechanismus nicht plausibel ist oder eine Erklärung ganz fehlt. Etwas anders stellt sich die Situation bei einer bekannten Knochenerkrankung dar. Der Nachweis einer 
Fraktur allein stellt keinen Verdachtsfall dar, wohl aber eine Häufung von Frakturen oder z. B. ein an Leugnung der Erkrankung grenzender Umgang der Eltern mit dem Kind. Hierbei ist zu beachten, dass auch Kinder mit einer chronischen Grunderkrankung Opfer einer Kindesmisshandlung werden können. Umgekehrt betrachtet wird bei einem Misshandlungsverdacht häufig von Eltern oder anderen Beteiligten eine „Knochenerkrankung“ als Erklärung für ansonsten unerklärliche Frakturen und als Gegenargument zu einer Kindesmisshandlung angeführt.

\section{Primäre und sekundäre Osteoporose}

Um die im Zusammenhang mit unklaren Frakturen immer wieder auftretende Differenzialdiagnose einer pathologischen Fraktur oder einer unbekannten Grunderkrankung mit einer verringerten Stabilität des Skelettsystems überprüfen zu können, ist es notwendig, die Schritte der physiologischen Knochenentwicklung zu kennen. Die Muskeln sind verantwortlich für den Antrieb und stellen das kontraktile Element der Bewegung dar. Knochen geben den stabilen Rahmen vor, an dem die Muskeln fixiert sind, um ihre Funktion zu erfüllen. Die Muskelaktivität wird durch das zentrale und periphere Nervensystem kontrolliert. Es ist bekannt, dass Nerven, Muskeln und Knochen eine funktionelle Einheit darstellen [3].

Bereits 1892 beschrieb der deutsche Anatom Julius Wolff den Zusammenhang zwischen Muskeln und Skelettentwicklung in seinem „Gesetz der Transformation der Knochen“ [4]. Dieses Gesetz beschreibt, dass sich die Architektur des Skelettsystems an die auf es wirkenden Kräfte (Anforderungen) anpasst, nämlich die maximalen Kräfte, die von den Muskeln ausgeübt werden. Daraus ergibt sich, dass die Knochenarchitektur mit Kortikalis und Spongiosa eine Folge der Nutzung des Skelettsystems ist. Körperliche Bewegung fördert die Aktivität von Osteoblasten und dementsprechend die Knochenbildung, während Immobilität zum Knochenabbau führt.

Wenn über die Muskulatur ein ausreichender osteoanaboler Stimulus ausgeübt wird, reagieren die Osteoblasten mit einer vermehrten Produktion von Kollagen (auch als Osteoid bezeichnet). Dieses Kollagen wird aus der Zelle ausgeschleust und bildet einen Bestandteil der extrazellulären Matrix. In einem zweiten, davon zunächst unabhängigen Schritt erfolgt dann die Einlagerung von Kalzium und Phosphat in das Osteoid. Synthese und Mineralisierung sind also 2 unterschiedliche Schritte, die differenzialdiagnostisch unterschieden werden müssen. Eine fehlende Synthese kann die Folge einer reduzierten Muskelaktivität (Immobilisationsosteoporose) sein oder einer Funktionsstörung der Osteoblasten (angeborene Skeletterkrankungen wie Osteogenesis imperfecta). Therapeutisch ist eine Kräftigung der Muskulatur erforderlich, und es kann bereits bei geringeren Krafteinwirkungen zu
Frakturen kommen. Im Gegensatz hierzu handelt es sich bei Störungen der Mineralisierung fast immer um einen durch Ernährung und Lebensstil verursachten Mangel an Substraten, der zu einer geringeren Knochenstabilität führt. Hier muss im Sinne der Kindeswohlgefährdung eine Vernachlässigung geprüft werden.

Neben der Regulation des Knochen- und des Muskelwachstums durch biomechanische Einflüsse stellten sich weitere Faktoren als relevant heraus [5]. In dem Regelkreis der funktionellen Muskel-Knochen-Einheit werden die mechanischen Faktoren (Maximalkräfte der Muskulatur) von den nicht mechanischen Einflüssen (Hormone, Nahrungsbestandteile und Medikamente) getrennt. Diese nicht mechanischen Einflüsse haben eine modifizierende Wirkung auf den Regelkreis und beeinflussen z.B. die Osteoblastenaktivität durch Parathormon oder Testosteron, die Osteoklastentätigkeit durch Osteoprotegerin und die Osteozytenempfindlichkeit möglicherweise durch Östrogen. Diese Einflüsse können jedoch die mechanischen Kräfte auf den Knochen durch die Muskulatur nicht ersetzen, sondern nur modifizieren.

Wie oben dargestellt, ist die Knochenfestigkeit abhängig von den einwirkenden Kräften durch die Muskulatur. Dies hat sich im klinischen Alltag unter Berücksichtigung der funktionellen Muskel-Knochen-Einheit als guter Screeningparameter zur Differenzierung von angeborenen Störungen mit frühem Knochenverlust oder chronischen Erkrankungen mit verringerter Muskelaktivität bewährt. Es wird ein zweistufiges Verfahren zur Diagnostik empfohlen, welches z. B. auf die bei einer DXA-Messung erhobener Daten (s.u.) angewendet werden kann. Im ersten Schritt wird die Muskelentwicklung unter Berücksichtigung der Körperlänge untersucht. Im zweiten Schritt wird die Skelettadaption an die Muskelentwicklung analysiert. Durch dieses Vorgehen können primäre und sekundäre Skeletterkrankungen unterschieden werden [6].

Unabhängig von primärer oder sekundärer Entstehung der Skeletterkrankung haben diese ein erhöhtes Frakturrisiko gemeinsam. Hinzu kommt bei Inaktivität die Störung der Körperkoordination, welche ebenfalls das Sturzrisiko und somit das Frakturrisiko erhöht. Der Sturz ist der größte Risikofaktor für eine Fraktur und ein erhöhtes Frakturrisiko ein limitierender Faktor für die Lebensqualität und Patientensicherheit.

\section{Diagnostische Schritte bei einer Fraktur unklarer Genese}

\section{Anamnese}

Eine gründliche Anamnese ist wichtiger Bestandteil der Diagnostik. Es muss die Plausibilität des Unfallhergangs geprüft und hinterfragt werden, ob das Kind überhaupt in der Lage ist, den beschriebenen Unfallmechanismus 


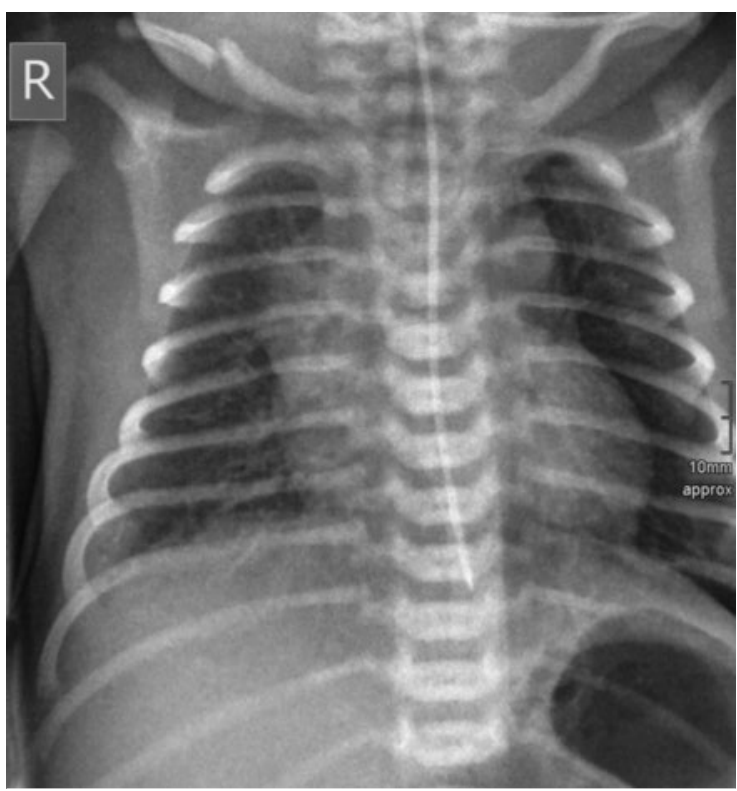

- Abb. 1 Klavikulafraktur nach Spontanpartus als geburtstraumatische Komplikation.

herbeizuführen. Beispielsweise sind Misshandlungen bei prämobilen Säuglingen mit Frakturen sehr wahrscheinlich. Ein Kind, das sich von der motorischen Entwicklung her noch nicht drehen kann, wird kaum selbstständig vom Wickeltisch fallen. Ein Geburtstrauma sollte ausgeschlossen werden, kommt aber immer bei Verletzungen in den ersten Lebenstagen differenzialdiagnostisch in Betracht und muss z. B. bei einer Klavikulafraktur erwogen werden ( $\mathbf{A} \mathbf{b b}$. 1) [7] sowie nach Entbindung per Sectio bei Kantenabsprengungen der distalen Tibiae.

Merke

Der Verdacht auf eine Kindesmisshandlung entsteht häufig, wenn die Anamnese für den vorliegenden Befund nicht plausibel erscheint.

Einige Eltern geben bei der Anamneseerhebung an, dass es keinen besonderen Vorfall gegeben habe und dass das Kind plötzlich das Gehen verweigere oder eine Extremität nicht bewegen wolle. Dies ist bei einem knochengesunden Kind unwahrscheinlich und sollte immer eine umfassende Diagnostik nach sich ziehen. Allerdings müssen anamnestisch Hinweise auf eine Knochenerkrankung erfasst werden. Es sollte erfragt werden, ob in der Vergangenheit häufiger Frakturen ohne adäquates Trauma auftraten oder ob eine positive Familienanamnese für skelettale Erkrankungen, Muskelerkrankungen oder Blutgerinnungsstörungen vorliegt. Weiterhin sollte im Vorsorgeheft überprüft werden, ob es in den Vorsorgeuntersuchungen Hinweise auf eine systemische Skeletterkrankung, Mineralisierungsstörung des Knochens oder motorische Auffälligkeiten gibt. Eine Gedeihstörung oder ein
Entwicklungsknick (hierbei ist der Perzentilenverlauf relevant bzw. ein Perzentilen kreuzendes Wachstumsverhalten auffällig) sollten ausgeschlossen werden.

Wichtig ist weiterhin die Berücksichtigung von sekundären Knochenerkrankungen im Rahmen von malignen Erkrankungen, Medikamenteneinnahme (z. B. Cortison), Infektionen (z. B. Osteomyelitis), Immobilität oder Mangelernährung, wobei Letzteres auch Folge einer schweren Vernachlässigung sein kann und somit eine Form der Kindesmisshandlung darstellt. Auch die Aussagen des betroffenen Kindes können auf eine Misshandlung hinweisen. Die Befragung des Kindes muss altersgerecht erfolgen und eine zusätzliche Traumatisierung ist zu vermeiden. Zur vollständigen Anamnese gehört auch eine Sozial- und Familienanamnese: Gibt es Hinweise auf eine belastete Eltern-Kind-Beziehung oder eine Überforderung der Eltern mit dem Kind/den Kindern? Befindet sich die Familie in einer aktuellen Krisensituation? Welche Personen leben sonst noch mit im Haushalt des Kindes?

Der Zeitpunkt der klinischen Vorstellung bei schweren Verletzungen muss beachtet werden: Gibt es eine zeitliche Verzögerung zwischen Unfallhergang und Vorstellung bei der Ärztin bzw. beim Arzt? Gibt es Differenzen zwischen den Aussagen der einzelnen Angehörigen? Werden wechselnde Erklärungsmuster für vorliegende Verletzung benutzt? Die Anzahl der Frakturen ist ebenso wichtig wie die Lokalisation und der Verlauf der Frakturlinie. Rezidivierende unklare Verletzungen und das häufige Wechseln der medizinischen Betreuung stellen Hinweise auf eine mögliche Kindesmisshandlung dar. Man sollte nach Begleitverletzungen des Kindes schauen und diese in der körperlichen Untersuchung ausschließen. Wenn sich anamnestische Hinweise für frühere medizinische Behandlungen in anderen Krankenhäusern finden, sollte versucht werden, eine Schweigepflichtentbindung der Eltern zu erhalten, um dort erhobene Befunde einzusehen.

\section{Untersuchung}

Bei der körperlichen Untersuchung sollten zunächst die Vitalzeichen und dann der Frakturverdacht geprüft werden. Dabei sind die periphere Durchblutung, Motorik und Sensibilität zu untersuchen. Wichtig ist es, das Wohlergehen des Kindes während der gesamten Untersuchung zu sichern und keine weiteren Schmerzen zu verursachen. Ggf. ist während der Untersuchung eine Analgesie einzuleiten, und es sollte versucht werden, das Kind möglichst wenig zu bewegen. Die Prüfung der Krepitation, wie manchmal noch zur Frakturdiagnostik empfohlen, ist unbedingt zu vermeiden. Die einzelnen Schritte der Untersuchung müssen dem Kind erklärt werden, um eine weitere Traumatisierung zu verhindern. Weitere ossäre Verletzungen müssen ausgeschlossen werden, dazu zählen beispielsweise Frakturen anderer Extremitäten, der Wirbelsäule oder auch Rippenfrakturen. Ggf. ist bei 


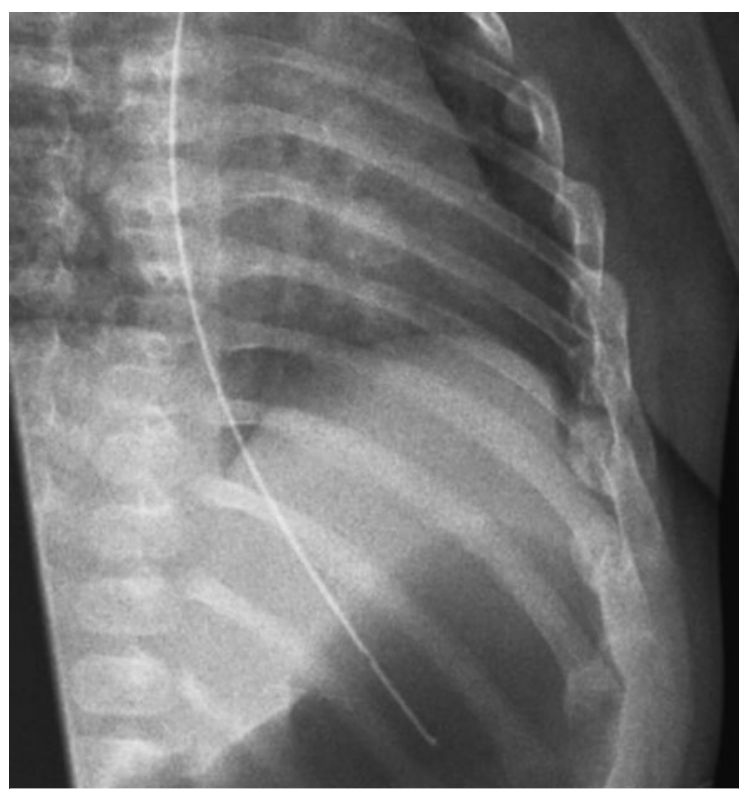

Abb. 2 Röntgenaufnahme des Thorax eines 3 Monate alten intubierten Säuglings mit einer linksseitigen Rippenserienfraktur.

den möglicherweise erforderlichen aufwendigen radiologischen Maßnahmen eine Sedierung zu erwägen.

Bei verschiedenen Frakturen gibt es bestimmte wahrscheinliche oder unwahrscheinliche Entstehungsmechanismen. Besonders Rippenserienfrakturen sind nahezu pathognomonisch für eine Misshandlung. Bei einer kardiopulmonalen Reanimation oder einem schweren Verkehrsunfall auftretende Rippenfrakturen sind selten und lassen sich anamnestisch klar erkennen. Sie sollten kein Problem in der Abgrenzung zu einer Kindesmisshandlung darstellen ( $\triangleright$ Abb. 2). Bei komplexen Schädelfrakturen ist eine Misshandlung als Ursache ebenfalls wahrscheinlich, da solche nur durch eine erhebliche Gewalteinwirkung entstehen können und die unfallbedingte Entstehung auch anamnestisch meist klar zu erfassen ist.

An die symptomorientierte Untersuchung muss eine sorgfältige Untersuchung der Haut angeschlossen werden. Diese kann wichtige Hinweise auf eine mögliche Misshandlung liefern. Hämatome an Prädilektionsstellen wie den Ohren, der behaarten Kopfhaut (über der „Hutkrempenlinie“), der Mundschleimhaut, dem Gesäß oder den Genitalien weisen ebenso auf eine mögliche Misshandlung hin wie bestimmte geformte Hämatome, die auf eine Misshandlung mit einem Gegenstand hinweisen können ( $\triangleright$ Abb. 3). Hämatome und Frakturen weisen dabei eine zu vernachlässigende Korrelation auf [8]. Hinweise, bei welcher Art von Hämatomen eine weitere Untersuchung erforderlich ist, gibt die „Kinderschutz-Leitlinie“ [9]. Abwehrverletzungen, z. B. an den Parierflächen der Arme, sollten ebenfalls beachtet werden.

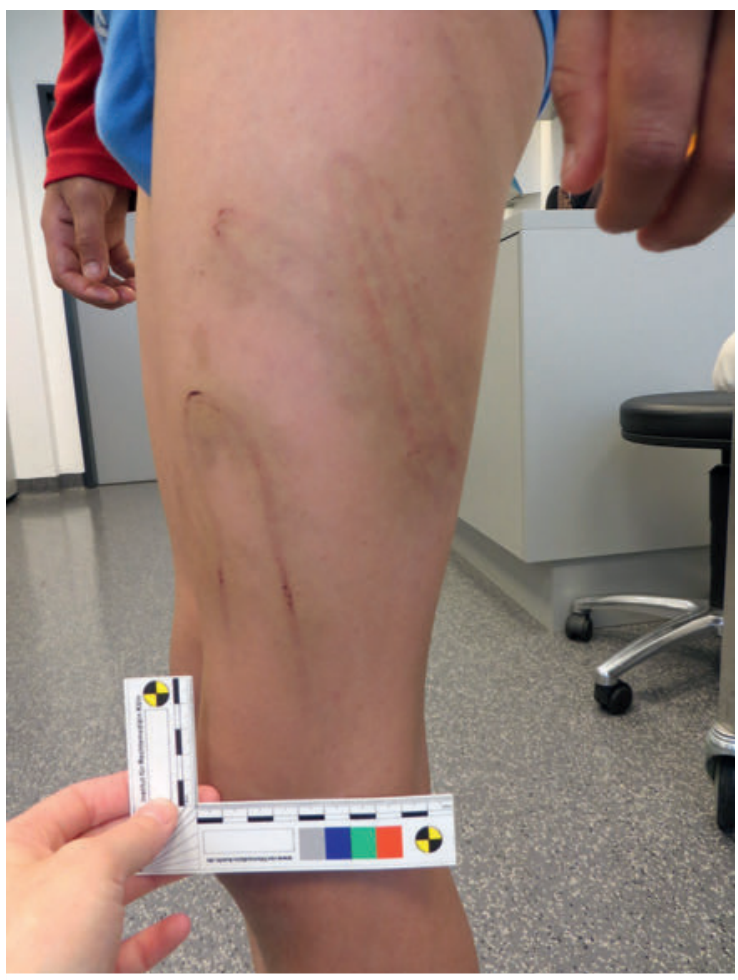

- Abb. 3 Fotografie des rechten Beines eines Jungen mit Hämatomen durch Schläge mit einem zur Schlaufe gelegten Seil. Diese geformten Verletzungen fanden sich auch anderen Lokalisationen (Hals, Arme). Unfallbedingte Stürze können die Formung, Lage und Ausrichtung nicht erklären.

Der Gesamteindruck des Kindes, der Ernährungsstand sowie der Pflegezustand sollen berücksichtigt und dokumentiert werden. Skelettale Auffälligkeiten, die auf eine Skelettdysplasie hinweisen, oder auch syndromale Erscheinungen können den Verdacht auf eine Misshandlung relativieren. Dazu zählen beispielsweise Frakturen und Deformierungen der langen Röhrenknochen, eine Schwäche des Bandapparats oder ein Kleinwuchs.

\section{Dokumentation}

Eine genaue, juristisch verwertbare, klar leserliche Dokumentation ist unabdingbar bei Verdacht auf Kindesmisshandlung. Dabei sollten Aussagen möglichst wortgetreu dokumentiert werden. Die Situation, in der Aussagen getroffen werden (z. B. eines Kindes in Abwesenheit der Eltern), muss aus der Dokumentation entnommen werden können. Subjektive Eindrücke gehören ebenso dokumentiert wie Telefonate, Austausch mit anderen Fachabteilungen oder der niedergelassenen Kinderärztin bzw. dem niedergelassenen Kinderarzt. Die Dokumentation dient nicht nur als Beweismaterial, wenn ein Strafverfahren eingeleitet werden sollte, sondern auch der eigenen Erinnerung und Absicherung (,was nicht dokumentiert ist, ist nicht gemacht“). Die körperliche Untersuchung sollte ebenso festgehalten werden wie die Anamnese. 


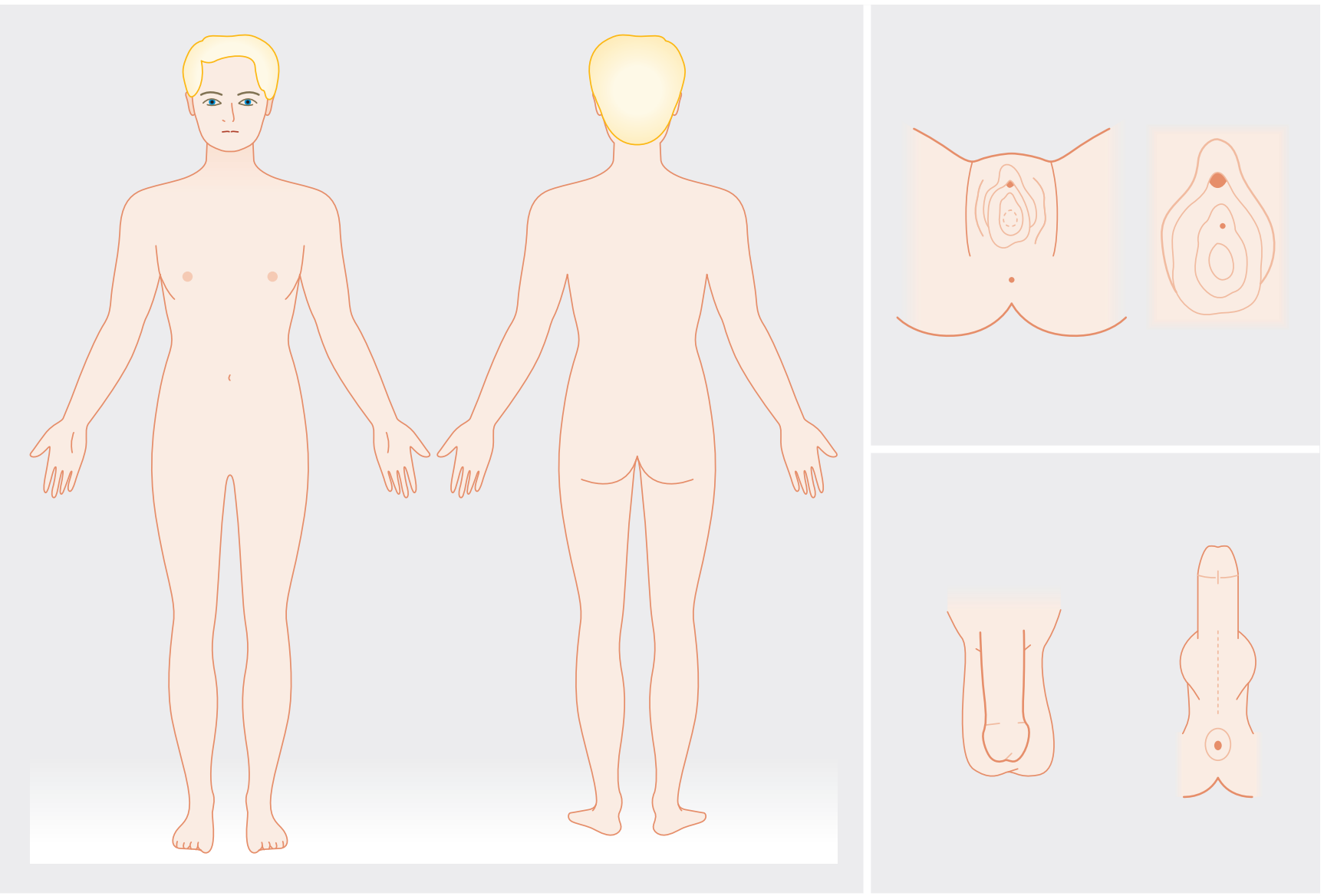

- Abb. 4 Skizzierungsvorlagen zur Dokumentation von Verletzungen bei einem Verdacht auf Kindesmisshandlung.

Neben der Skizzierung der Verletzungsorte sollte eine Fotodokumentation äußerlicher Verletzungen mit Maßstab erfolgen, wobei entsprechende datenschutzrechtliche Belange beachtet werden müssen. Hierbei können strukturierte Dokumentationsmappen und Abbildungen hilfreich sein, um eine umfassende Dokumentation zu gewährleisten (॰ Abb. 4).

\section{Bildgebende Verfahren}

Bei einem dringenden Verdacht auf eine skelettale Verletzung ist eine entsprechende bildgebende Diagnostik notwendig, wobei beachtet werden muss, dass die von Kindern angegebene Lokalisation nicht immer mit der Stelle der Verletzung übereinstimmt. Deshalb müssen auch angrenzende Bereiche bildgebend dargestellt werden, um z. B. eine Verletzung am Femur nicht zu übersehen, wenn das Kind Schmerzen am Unterschenkel angibt.

Merke

Die wichtigste diagnostische Maßnahme neben der Anamneseerhebung und der klinischen Untersuchung ist die Durchführung von Röntgenaufnahmen. Sie dient sowohl zur Diagnose der Fraktur als auch zur Suche nach anderen, mehrzeitigen Verletzungen.
Zur bildgebenden Diagnostik bei Verdacht auf eine Fraktur ist die konventionelle Röntgendiagnostik das Mittel der Wahl. Neben der Fraktur muss auf dem Röntgenbild auch nach anderen Auffälligkeiten gesucht werden, die eine Erklärung für die Verletzung liefern können. Hierzu können lokale Knochendestruktionen wie bei einem Tumor oder einer Knochenzyste bei fibröser Dysplasie gehören. Es sollte auch eine Beurteilung der Knochenstruktur in Hinblick auf Kortikalis und Spongiosa erfolgen. Hier können Hinweise auf Störungen der Osteoklasten- oder Osteoblastenfunktion gefunden werden oder sich Veränderungen der gesamten Architektur der Röhrenknochen zeigen. So können Röhrenknochen mit einem geringen diaphysären Durchmesser z. B. auf eine langandauernde Muskelerkrankung oder Immobilität hindeuten. Bei Patienten mit einer Spina bifida und einem hoch-lumbalen Läsionsort finden sich oft lange und dünne Röhrenknochen, bei denen es schon bei geringen Krafteinwirkungen zu Frakturen kommen kann.

Merke

Differenzialdiagnostisch sollten angeborene Skelettdysplasien, Mineralisierungsstörungen oder andere systemische Erkrankungen ausgeschlossen werden, die das muskuloskelettale System beeinflussen. 
Sollten sich auf dem Bild ein untypischer Verlauf der Frakturlinien oder andere Hinweise für eine Kindesmisshandlung zeigen, dann ist eine weiterführende radiologische Diagnostik erforderlich, um ältere oder klinisch nicht offensichtliche Verletzungsfolgen zu erkennen. Hierzu gehört ein standardisiertes Röntgen-Skelettscreening. Dieses setzt sich wie folgt zusammen ( $\triangleright$ Abb. 5):

- Schädel a.-p. und seitlich

- Thorax a.-p.

- Oberarm a.-p. beidseits

- Unterarm a.-p. beidseits

- Hand p.-a. beidseits

- Oberschenkel a.-p. beidseits
- Unterschenkel a.-p. beidseits

- Fuß a.-p. beidseits

\section{Merke}

Diagnostisch ist das Röntgen-Skelettscreening Mittel der Wahl. Eine Knochenszintigrafie oder ein Babygramm werden nicht mehr durchgeführt.

Wenn sich der Verdacht bestätigt, sollten je nach vorliegenden Befunden weitere Röntgenuntersuchungen erfolgen:

- Thorax schräg links und Thorax schräg rechts (zum Nachweis einer Rippenfraktur)

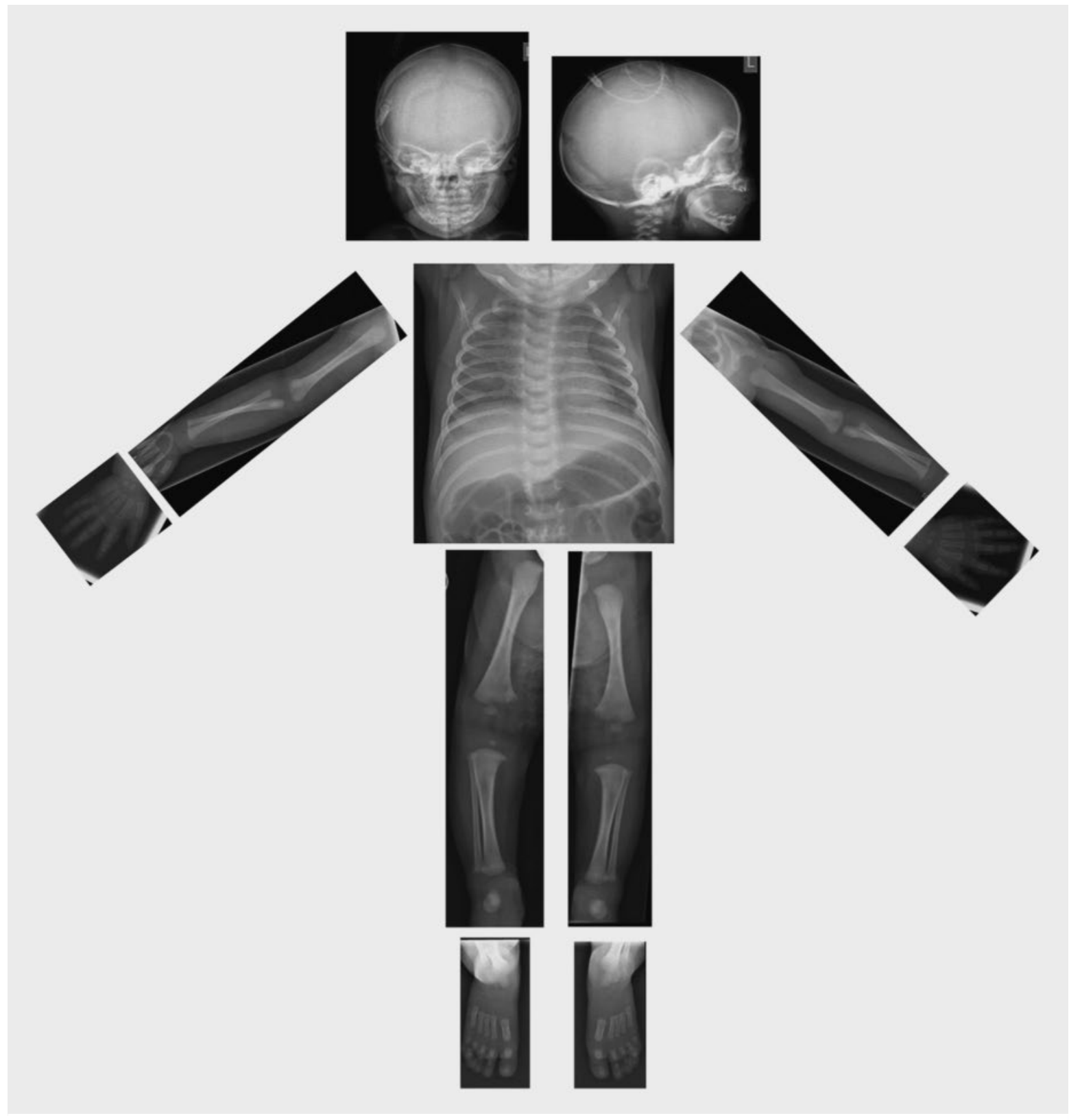

- Abb. 5 Erforderliche Röntgenaufnahmen für ein Skelettscreening bei Verdacht auf Kindesmisshandlung. 


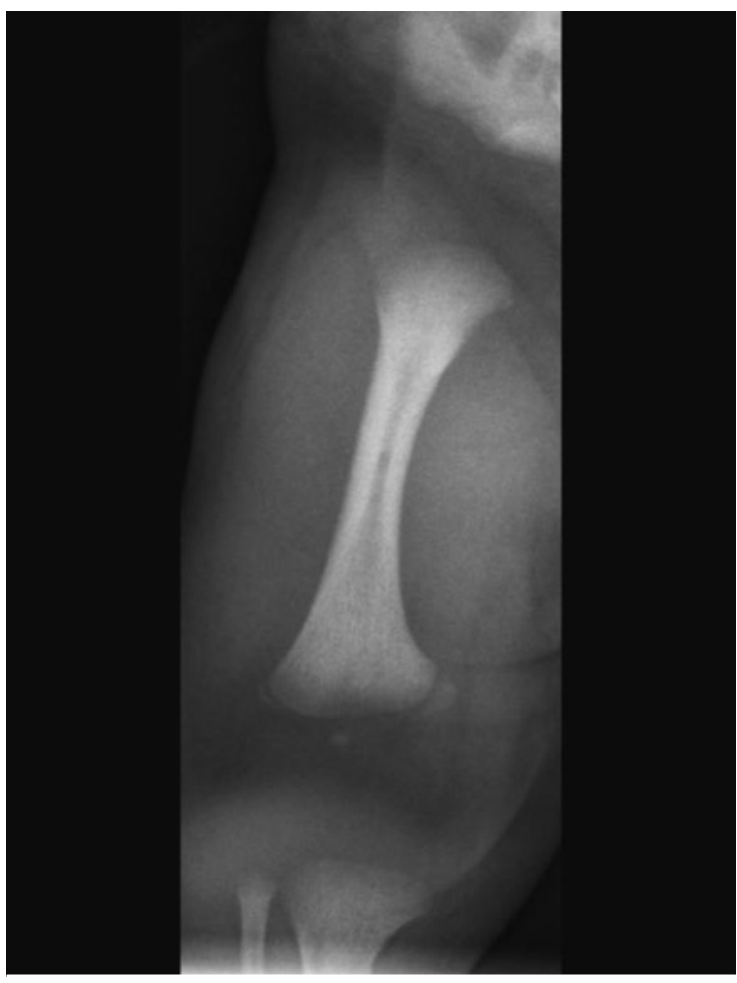

Abb. 6 Röntgenaufnahme des Femurs mit Korbhenkelfraktur. Quelle: Dr. Jörg Schaper, Universitätsklinikum Düsseldorf

- Wirbelsäule seitlich (bei Vorliegen multipler Frakturen oder V.a. Skelettdysplasie)

- Hüfte und Becken a.-p.

Wenn im initialen Röntgen-Skelettscreening kein Nachweis einer Fraktur vorliegt, der Verdacht auf eine Misshandlung mit skelettalen Folgen jedoch weiterhin besteht, sollte das Screening nach 11-14 Tagen wiederholt werden [9]. Ein Geschwisterscreening ist zu beachten. Bei unklaren Befunden empfiehlt es sich, eine kinderradiologische Zweitmeinung einzuholen, welche die Bilder unabhängig von der ersten radiologischen Einschätzung bewertet. Dabei gilt es typische Verletzungsmuster als Misshandlungsfolgen zu identifizieren $[10,11]$. Multiple Frakturen in verschiedenen Heilungsstadien, metaphysäre Frakturen der langen Röhrenknochen, Rippenfrakturen und komplizierte Schädelfrakturen ohne passende akzidentelle Anamnese gelten als pathognomonisch für eine Kindesmisshandlung. Ebenso sind metaphysäre Frakturen (Eckfrakturen oder Korbhenkelfrakturen, > Abb. 6) beim kleinen Kind hochspezifisch für eine nicht akzidentelle Ursache $[12,13]$.

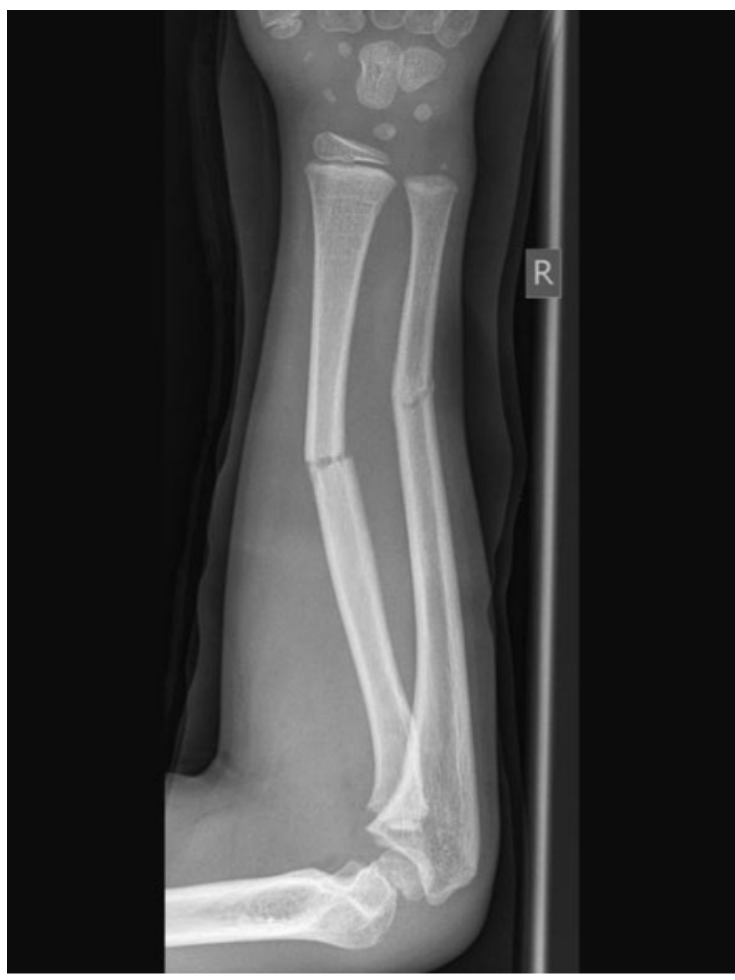

Abb. 7 Röntgenbild eines Unterarms mit Hand- und Ellenbogengelenk nach Verkehrsunfall. Es zeigt sich eine diaphysäre Fraktur von Radius und Ulna. Der Unfallhergang stellt eine plausible Erklärung für die Verletzung dar. Ein ähnliches Aussehen kann auch eine Parierfraktur als Abwehrverletzung bei einer Kindesmisshandlung aufweisen.

\section{Merke}

Multiple Frakturen in verschiedenen Heilungsstadien, metaphysäre Frakturen der langen Röhrenknochen und Rippenserienfrakturen machen eine Misshandlung als Ursache wahrscheinlich, wohingegen Wirbelkörperfrakturen eher auf eine systemische Skeletterkrankung hindeuten.

Bei prämobilen Säuglingen können durch Rotations- und Scherkräfte sog. periostale Reaktionen entstehen. Dabei kommt es zu einer Ablösung und Unterblutung des Periosts, welches metaphysär am Knochen fixiert ist und sich diaphysär abheben kann. Diaphysäre Frakturen stellen eine differenzialdiagnostische Herausforderung dar, da sie oft akzidentell-traumatisch bedingt sind, sie können aber auch im Zusammenhang mit einer Kindesmisshandlung auftreten. Hier muss besonders sorgfältig die Plausibilität des Unfalls überprüft werden. Dazu zählt auch das Alter des Kindes, da bei prämobilen Kindern eine Misshandlung als Ursache einer diaphysären Fraktur wahrscheinlich ist. 
Bei älteren Kindern ist besonders bei Tibia- oder Unterarmfrakturen eine gründliche Prüfung erforderlich, da hier eine Parierfraktur vorkommen kann, die häufig beim Versuch der Abwehr entsteht und nur anamnestisch und nicht aufgrund der Bildgebung von einer akzidentelltraumatischen Fraktur unterschieden werden kann ( $\vee$ Abb. 7). Frakturen an Humerus oder Femur erfordern eine hohe Krafteinwirkung und können deshalb meist durch die Anamnese von einer Kindesmisshandlung abgegrenzt werden ( $\triangleright$ Abb. 8). Differenzialdiagnostisch sind akzidentelle Frakturen wie die „Toddler's fracture“ (Spiral- oder Schrägfraktur meist im Bereich der distalen Drittel der unteren Extremität) oder geburtstraumatische Frakturen in Betracht zu ziehen, wie sie z. B. häufig an der Klavikula vorkommen können ( $\bullet$ Abb. 1).

Wenn der Verdacht auf eine misshandlungsbedingte Schädel-Hirn-Verletzung vorliegt (beispielsweise bei schweren intrakraniellen Läsionen nach einem angeblich banalen Sturzereignis), muss zunächst eingeschätzt werden, ob das Kind vital bedroht ist. Wenn keine vitale Bedrohung vorliegt, sollte eine MRT des Schädels einer CTUntersuchung vorgezogen werden (eine alleinige Sonografie des Schädels ist nicht ausreichend). Bei hochgradigem Verdacht auf weitere Verletzungen sollte in diesem Zusammenhang möglichst auch eine Darstellung der ganzen Wirbelsäule erfolgen.

Die Durchführung einer Skelettszintigrafie zur weiteren Frakturlokalisation ist wegen der schlechten räumlichen Auflösung, der physiologischen Anreicherung im Bereich der Wachstumsfugen und der Strahlenbelastung obsolet. Auch Ganzkörper-MRT-Untersuchungen können ein konventionelles Röntgenscreening nicht ersetzen, da metaphysäre Frakturen seltener erkannt werden [14]. Ein Babygramm sollte aufgrund der hohen Strahlenbelastung ebenfalls nicht mehr durchgeführt und durch gezielte Einzelaufnahmen (s.o.) ersetzt werden.

\section{FALLBEISPIEL 1}

Es erfolgte die Vorstellung eines 6 Monate alten männlichen Säuglings in der Notaufnahme einer Kinderklinik aufgrund einer Rötung und Schwellung des rechten Oberarms. Laut Mutter sei aufgefallen, dass er den Arm nicht mehr bewegen wolle. Als sie den Arm zu Hause genauer untersucht habe, sei ihr die Schwellung aufgefallen. Sie habe keinen Unfall beobachtet, allerdings stecke er oft den Arm durch das Gitterbett. Sie könne sich vorstellen, dass er sich die Verletzung dadurch zugezogen habe. Der Junge sei ansonsten gesund, gut gediehen, sie habe alle Vorsorgetermine wahrgenommen und alle Impfungen wurden nach den Empfehlungen der STIKO durchgeführt. Es seien bisher keine Krankenhausaufenthalte erforderlich gewesen und auch keine chronischen Erkrankungen bekannt. Die Eltern leben getrennt, die Mutter wohne alleine mit dem Sohn und seiner 5-jährigen Schwester.

In der Notaufnahme zeigt sich ein gut entwickelter, freundlich zugewandter Säugling in sauberer Kleidung. Das Körpergewicht liegt auf der 50. Perzentile, die Körperlängenentwicklung lediglich auf der 10. Perzentile. In der Beobachtung der Spontanmotorik zeigt sich eine verminderte Aktivität des rechten Arms. Beim Ausziehen scheint der Säugling Schmerzen zu verspüren und beginnt zu weinen. Der rechte Oberarm ist gerötet, sein Umfang im Vergleich zur Gegenseite vergrößert. Kein Hinweis auf eine Durchblutungs-, Motorik- oder Sensibilitätsstörung. Insgesamt wirkt der Säugling bewegungsarm und der Muskeltonus hypoton. Die weitere körperliche Untersuchung erweist sich als unauffällig.

In der Röntgenuntersuchung des rechten Oberarms a.-p. und axial zeigt sich eine lineare Frakturlinie in Schaftmitte. Außerdem sieht man eine deutliche Kallusbildung an derselben Stelle. Dies spricht für eine bereits in Heilung befindliche Fraktur. Die Kortikalis erscheint normal, wenngleich insgesamt der Knochen etwas strahlentransparent erscheint. Aufgrund der fragwürdigen
Anamnese und dem vorliegenden Röntgenbefund erfolgte die stationäre Aufnahme des Säuglings zur Behandlung und weiteren diagnostischen Abklärung. Die Fraktur wurde am selben Abend konservativ behandelt und ruhiggestellt.

Beim V.a. das Vorliegen einer Kindesmisshandlung wurde nach Rücksprache und Mitbeurteilung durch die Kollegen aus der Kinderschutzgruppe einschließlich der Rechtsmedizin ein RöntgenSkelettscreening eingeleitet. Dabei erkannte man am linken Humerusschaft ebenfalls eine Kallusbildung, ansonsten keine weiteren Auffälligkeiten. Auch die Ultraschalluntersuchung des Schädels erbrachte einen Normalbefund. Die augenärztliche Untersuchung zeigte keine Besonderheiten. Im Blutbild zeigten sich bis auf eine leichte Leukozytose keine Auffälligkeiten. Das Labor zur Bestimmung der Knochenstoffwechselparameter erbrachte eine auf das 1,5-Fache der Norm erhöhte alkalische Phosphatase und einen leicht erniedrigten Vitamin-D-Spiegel. Die Kalziumund Phosphatausscheidung im Urin waren normwertig.

Es folgte eine multidisziplinäre Besprechung der Kinderschutzgruppe. Dabei wurde entschieden, ein klärendes Gespräch mit der Mutter zu suchen und den Verdacht auf eine Kindesmisshandlung im Beisein des Psychosozialdienstes und des Jugendamtes zu äußern. Die Mutter brach während des Gesprächs in Tränen aus und gab zu, etwas „forscher“ zuzugreifen, wenn der Junge nicht aufhöre zu weinen. Auch könne es sein, dass sie die regelmäßige Vitamin-D-Prophylaxe nicht immer konsequent verabreicht habe. Sie habe nie die Absicht gehabt, ihrem Kind Verletzungen zuzufügen, wisse in solchen Augenblicken allerdings nicht weiter. Das Jugendamt besuchte die Mutter zu Hause und organisierte eine Familienhilfe, die die Mutter unterstützen soll. Unter diesen Voraussetzungen und mit der Maßgabe regelmäßiger Vorstellungen des Kindes bei der betreuenden Kinderärztin wurde der Säugling in Absprache mit dem Jugendamt in das häusliche Umfeld entlassen. 


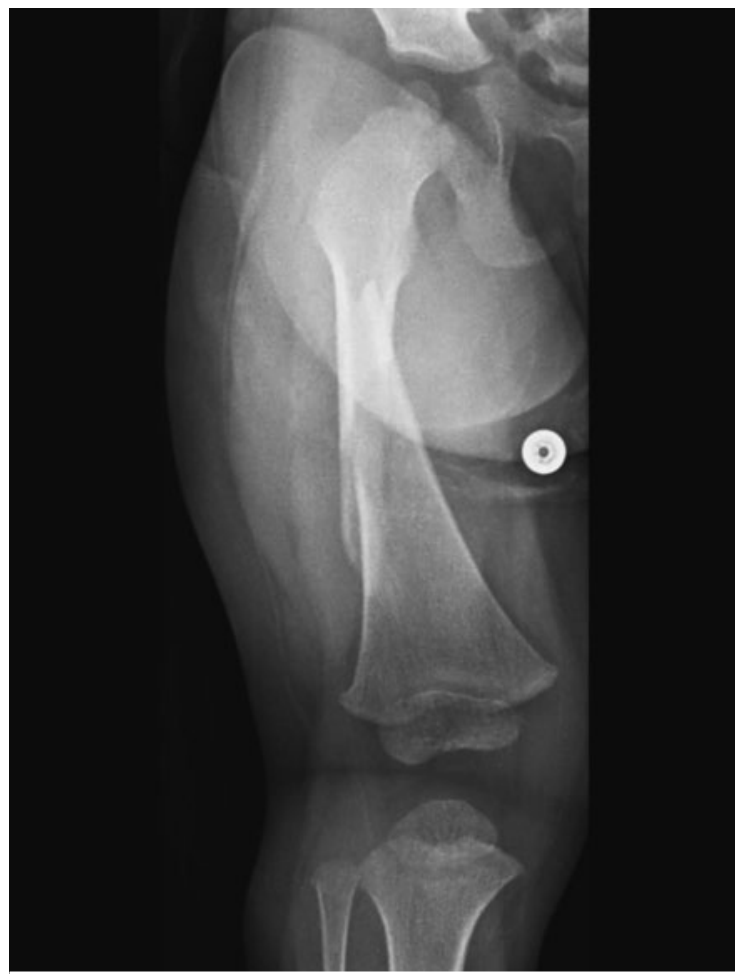

- Abb. 8 Röntgenbild eines 8 Monate alten Kindes mit Femurschaftfraktur. Die Vorstellung erfolgte aufgrund einer Schwellung des Oberschenkels, anamnestisch sei ein Trauma nicht erinnerlich.

\section{Labor}

Bei einer Fraktur muss immer durch Laboruntersuchungen überprüft werden, ob es eine systemische oder syndromale Ursache für eine erhöhte Frakturneigung gibt. Die Laboruntersuchungen dienen auch dazu, zwischen Mineralisierungs-, Synthese- und Resorptionsstörungen zu unterscheiden. Diese Unterscheidung ist entscheidend, um zu erkennen, in welchem Abschnitt der physio- logischen Skelettentwicklung die Problematik liegt oder ob es keinen Hinweis für eine systemische Ursache gibt, welche die Fraktur erklären könnte.

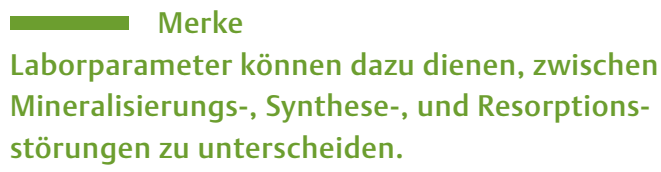

Meist ist die Bestimmung von 5 Parametern ausreichend, um einen orientierenden Überblick über den Knochenstoffwechsel zu gewinnen. Um die Versorgung des Skelettsystems mit Substrat zum Knochenaufbau zu überprüfen, sollten die Serumwerte von Kalzium und Phosphat gemessen werden. Die Abschätzung, ob der Einbau der Mineralien in den Knochen normal verläuft, lässt sich durch die Bestimmung der Aktivität der alkalischen Phosphatase (AP), unter Berücksichtigung der altersspezifischen Normwerte, durchführen [15]. Hierbei muss beachtet werden, dass die AP bei einer Frakturheilung erhöht ist. Zur Evaluierung der Auf- und Umbauvorgänge dient die Bestimmung von Parathormon und Vitamin D.

- Tab. 1 gibt an, auf welche Erkrankungen Veränderungen dieses pädiatrisch-osteologischen Basislabors hinweisen können. Hierbei muss hervorgehoben werden, dass bei Skelettdysplasien und angeborenen Synthesestörungen häufig weitgehend unauffällige Laborbefunde zu finden sind. Normwertige Laboruntersuchungen bei erhöhter Anzahl von Frakturen sollten deshalb immer Anlass zur weiterführenden Diagnostik sein, wohingegen bei auffälligen Laborkonstellationen häufig die Diagnosestellung einfach ist und eine gezielte Therapie eingeleitet werden kann. Allerdings gilt auch hier zu beachten, dass eine systemische Skeletterkrankung eine Kindesmisshandlung nicht ausschließt (s. o.).

Zusätzlich sollte ein Blutbild angefertigt werden, da auch andere systemische Erkrankungen wie Speicherkrankhei-

- Tab. 1 Typische Laborkonstellation bei erworbenen und angeborenen Skeletterkrankungen.

\begin{tabular}{|c|c|c|c|c|c|c|}
\hline & $\begin{array}{l}25-(\mathrm{OH}-) \mathrm{D}_{3} \\
\text { (ng/ml) }\end{array}$ & $\mathrm{Ca}$ & AP & $\mathrm{PO}_{4}$ & PTH & Metabolische Störung \\
\hline $\begin{array}{l}\text { Klinischer Vitamin-D-Mangel, } \\
\text { Rachitis }\end{array}$ & $<20$ & $\begin{array}{l}N L- \\
\downarrow\end{array}$ & $\uparrow \uparrow$ & $\begin{array}{l}N L- \\
\downarrow\end{array}$ & $\uparrow \uparrow$ & $\begin{array}{l}\text { Mineralisierungsstörung } \\
\text { (Substratmangel) }\end{array}$ \\
\hline hypophosphatämische Rachitis & NL & NL & $\uparrow$ & $\downarrow \downarrow$ & $\mathrm{NL}$ & $\begin{array}{l}\text { Mineralisierungsstörung } \\
\text { (Substratmangel) }\end{array}$ \\
\hline Hypophosphatasie & häufig $<20$ & $\uparrow=$ & $\downarrow \downarrow$ & $=\uparrow$ & $N L-\downarrow$ & $\begin{array}{l}\text { Mineralisierungsstörung } \\
\text { (Enzymdefekt) }\end{array}$ \\
\hline Osteogenesis imperfecta & $\mathrm{NL}$ & $\mathrm{NL}$ & NL $\uparrow$ & $\mathrm{NL}$ & $\mathrm{NL}$ & Synthesestörung \\
\hline Immobilisationsosteoporose & $\mathrm{NL}$ & NL $\uparrow$ & NL & NL $\uparrow$ & $N L-\downarrow$ & vermehrte Resorption \\
\hline \multicolumn{7}{|c|}{ AP: alkalische Phosphatase; NL: normal; PTH: Parathormon } \\
\hline
\end{tabular}


ten (Morbus Gaucher, Glykogenosen) oder hämatologische Erkrankungen (Sichelzellerkrankung, Leukämie) zu einer Beteiligung des Skelettsystems führen können (s. Übersicht weiter vorne).

\section{Knochendichtemessung}

\author{
Merke \\ Wenn durch radiologische Untersuchungen und \\ Laborbestimmungen keine ausreichende Erklärung \\ für das Auftreten von Frakturen gefunden wurde, \\ sollte eine Knochendichtemessung erfolgen.
}

Gleiches gilt, wenn sich bei der konventionellen radiologischen Untersuchung eine „osteopene Knochenstruktur“ als Hinweis auf eine reduzierte Knochenmasse zeigt. Die Methode der Knochendichtemessung muss in Abhängigkeit von Alter und der Fähigkeit des Kindes, während der Messung ruhig zu liegen, ausgesucht werden. Gibt es Hinweise auf eine unterschiedliche Knochenfestigkeit in verschiedenen Körperregionen, muss dies bei der Wahl der Messmethode ebenfalls berücksichtigt werden.

Am häufigsten verfügbar ist die DXA-Technik (Dual Energy X-ray Absorption) zur Bestimmung der Knochenflä- chendichte. Hierbei wird die zu untersuchende Region mit zwei Röntgenstrahlen unterschiedlicher Energie durchstrahlt. Aus der Absorption der Strahlung können die Art und Masse des untersuchten Gewebes berechnet werden. So kann zwischen Knochenmasse, Fettgewebe und „lean mass“ (ein Surrogatparameter für Muskelmasse) unterschieden werden. Wichtig im Kindesalter ist die Interpretation der Ergebnisse, die eine Anpassung nicht nur an das Alter, sondern auch an die Körpergröße und das Pubertätsstadium erfordert [16].

Bei der Analyse der DXA-Werte sollte nicht nur die Knochenmasse beurteilt werden, sondern auch die Muskelmasse. Zunächst sollte die Muskelmasse in Bezug zur Körpergröße evaluiert werden. Im zweiten Schritt erfolgt dann die Einbeziehung der Knochenflächendichte in das analytische Modell (s. o.). Hierbei muss die Körpergröße berücksichtigt werden, da sonst Kinder mit einem Kleinwuchs immer mit falsch niedriger Knochenmasse diagnostiziert werden $[3,17]$.

Bei Patientinnen und Patienten, bei denen die Muskelmasse im Vergleich zur Körpergröße erniedrigt ist, sind die auf den Knochen einwirkenden Kräfte - der osteoana-

\section{FALLBEISPIEL 2}

Es erfolgt die Vorstellung eines 15 Monate alten Mädchens, das laut Eltern bereits den gesamten Vormittag über schreie. Sie sei anders als sonst, sagt der Vater besorgt. Die Mutter sitzt weinend in der Notaufnahme und scheint sehr verzweifelt. Es sei immer ein unkompliziertes Kind gewesen, selten krank, trinke und esse gut. Es ist das erste Kind des jungen, entfernt verwandten Ehepaars. Das Vorsorgeuntersuchungsheft sowie der Impfpass sind altersentsprechend vollständig; Kopfgröße, Länge und Gewicht des Kindes liegen zwischen der 50. und 75. Perzentile.

Während der gesamten körperlichen Untersuchung weint das Kind und es bewegt sein linkes Bein kaum. Die Eltern haben dies nicht berichtet. Die restliche körperliche Untersuchung zeigt keine Auffälligkeiten. Die Frage, ob das Kind gestürzt oder ein anderes Trauma erinnerlich sei, wird verneint. Sie sei allerdings gestern bei ihrem Onkel gewesen, doch von einem Sturz o. ä. wurde nicht berichtet. Es wird eine Röntgenaufnahme des Ober- und Unterschenkels links a.-p. und seitlich durchgeführt, auf der sich eine nicht dislozierte Fraktur der proximalen Tibia zeigt. Der Femur erscheint verkürzt, insgesamt ist eine erhöhte Strahlentransparenz erkennbar. Die erneute Frage nach einem Trauma wird von den Eltern verneint. In der Familie sei die Mutter des Vaters an Brustkrebs erkrankt, ansonsten seien keine Vorerkrankungen bekannt. Die Fraktur wird daraufhin konservativ versorgt. Aufgrund der unklaren Situation wird das Kind stationär aufgenommen. Eine laborchemische Untersuchung zeigt außer einer erhöhten AP keinerlei Auffälligkeiten. Der Augenhintergrund erweist sich als unauffällig.
Nach Rücksprache mit der Kinderschutzgruppe samt Rechtsmedizin und Psychosozialdienst wird ein Röntgen-Skelettscreening durchgeführt und gleichzeitig eine molekulargenetische Untersuchung eingeleitet. Das Röntgen-Skelettscreening zeigt eine Kallusbildung auf Höhe der Schaftmitte des linken Humerus, der rechte Femur erscheint ebenfalls verkürzt, genauso wie der Humerus beidseits. Die erhöhte Strahlentransparenz zeigt sich im gesamten Röntgen-Skelettscreening. Die molekulargenetische Untersuchung erweist sich in Hinblick auf die Gene COL1A1 und COL1A2 (klassische Osteogenesis imperfecta) unauffällig. In der Urinuntersuchung kann eine erhöhte Osteoklastenaktivität nachgewiesen werden.

Da in der Anamnese glaubhaft keine Hinweise für Traumata zu eruieren sind, werden aufgrund der erhöhten Strahlentransparenz im Röntgen-Skelettscreening eine Knochendichtemessung und bei erniedrigten Werten eine erweiterte molekulargenetische Paneldiagnostik durchgeführt. Diese zeigt eine homozygote Mutation in dem Gen SERPINF1, die für eine OI-Typ 6 charakteristisch ist. Patienten mit dieser atypischen Form einer Ol entwickeln sich meist im 1. Lebensjahr normal, und es kommt erst ab dem 2. Lebensjahr zu progredienten Verbiegungen der Röhrenknochen und zu Frakturen. Der Befund wird ausführlich mit den Eltern besprochen, und es erfolgt eine Anbindung an eine spezialisierte Abteilung. 
bole Stimulus - zu gering und es kann deshalb keine ausreichende Knochenmasse aufgebaut werden. Dies findet sich z. B. bei Kindern mit SMA (spinaler Muskelatrophie). Ähnliches gilt bei Kindern, die aus anderen Gründen im Alltag auf die Nutzung eines Rollstuhls angewiesen sind.

Bei Kindern, bei denen die Knochenmasse im Verhältnis zur Muskelmasse reduziert ist, liegt eine primäre Osteoporose vor, da sich der Knochen nicht adäquat an die durch die Muskulatur ausgeübten Kräfte anpassen kann. Dies findet sich u.a. bei einer Osteogenesis imperfecta, bei der die Osteoblasten nicht ausreichend Knochensubstanz produzieren können, oder bei juveniler idiopathischer Osteoporose, bei der es zu einem vermehrten Knochenabbau kommt.

\section{Merke \\ Sind Muskelmasse und Knochenmasse für Alter und Größe adäquat, spricht dies gegen eine skelettale Erkrankung. Dies sollte dann weitere Abklärungen in Richtung einer Kindesmisshandlung begründen.}

Allerdings gibt es auch Sonderformen von Skeletterkrankungen, bei denen die Frakturgefahr erhöht, die Knochenflächendichtemessung aber unauffällig oder gar erhöht ist. Bei diesen Krankheitsbildern der Osteopetrose, der Osteosklerose oder der fibrösen Dysplasie finden sich meist auf dem konventionellen Röntgenbild eindeutige Hinweise.

\section{Merke \\ Besonders zur Diagnostik von Frühformen einer Skeletterkrankung ist die Untersuchung mittels peripherer quantitativer Computertomografie (pQCT) wegweisend.}

Diese Untersuchung erbringt eine physikalisch korrekte Bestimmung der Knochendichte (Masse/Volumen) und ermöglicht die differenzierte Beurteilung von Kortikalis, Spongiosa und Muskulatur [18]. Eine reduzierte Spongiosadichte kann hinweisend sein für eine sehr leichte Form einer Osteogenesis imperfecta oder auch als Form einer sekundären Osteoporose durch Medikamente (z. B. Cortison, Antikonvulsiva) auftreten [19].

\section{Molekulargenetik}

\author{
Merke \\ Sollte sich durch die o.g. Untersuchungen die Frage \\ einer angeborenen Skeletterkrankung nicht ein- \\ deutig klären, ist auch aus forensischen Gründen bei \\ Verdacht auf Kindesmisshandlung eine genetische \\ Untersuchung zu erwägen.
}

Hierbei ist es wichtig, eine klare Verdachtsdiagnose zu äußern oder mindestens dem Humangenetiker umfassende klinische Informationen zur Verfügung zu stellen, damit dieser eine zielgerichtete Analyse beginnen kann.
Die Eingrenzung der zu untersuchenden Gene wird u.a. durch die Nutzung sog. HPO-Terms (Human Phenotype Ontology) standardisiert, die eine immer bessere Interpretation der genetischen Befunde erlaubt. Meist werden inzwischen keine einzelnen Gene mehr evaluiert, sondern es erfolgt eine „Panel-Diagnostik“, bei der mehrere hundert Gene gleichzeitig untersucht werden können. Hierbei ist es allerdings von entscheidender Bedeutung, dass das richtige „Panel“ verwendet wird und nicht nur Gene untersucht werden, die einen Einfluss auf die Knochenbildung haben, wenn z. B. eine muskuläre Ursache vorliegt. Deshalb sind die vorherige Phänotypisierung und das Formulieren von Verdachtsdiagnosen entscheidend, um eine zielgerichtete genetische Untersuchung durchführen zu können [20].

\section{Weiteres Prozedere bei Vorliegen einer Kindesmisshandlung als Ursache der Fraktur}

Sollte sich aus den o.g. Untersuchungen oder der Anamnese der Verdacht ergeben, dass eine Misshandlung als Ursache der Fraktur in Frage kommt, muss das Kind stationär aufgenommen werden. Zum einen muss die akute Verletzung versorgt (konservativ versus operative Versorgung der Fraktur) und die Gesundheit des Kindes gewährleistet werden. Zum anderen wird somit der Kinderschutz gesichert und weitere Gefahren werden zunächst abgewendet, insbesondere dann, wenn die Situation unklar ist. Durch die stationäre Aufnahme kann die erforderliche Zeit gewonnen werden, um eine differenzialdiagnostische Abklärung einzuleiten und die nächsten Schritte zu planen. Hierzu gehört eine forensische Mitbeurteilung durch Rechtsmedizin, die jeweilige Kinderschutzgruppe, die Kinderradiologie und weitere im Einzelfall relevanten Disziplinen.

Merke
Bei einem Verdacht auf Kindesmisshandlung, ins-
besondere bei Hinweisen auf eine misshandlungs-
bedingte Schädel-Hirn-Verletzung, ist eine sorg-
fältige augenärztliche Untersuchung obligat.

Dabei steht die Frage nach retinalen Blutungen, wie sie oft im Zusammenhang mit einem Schütteltrauma auftreten, im Vordergrund. Die augenärztliche Untersuchung soll möglichst innerhalb der ersten 24 Stunden nach Vorstellung des Kindes erfolgen. Außerdem muss der körperlichen Untersuchung eine gründliche neurologische Untersuchung angeschlossen werden.

Falls der Verdacht auf zusätzliche viszerale Verletzungen vorliegt, muss eine Sonografie des Abdomens erfolgen oder ggf. eine weitere bildgebende Diagnostik. Wenn der Verdacht eines sexuellen Missbrauchs im Vorder- 
grund steht, sollte eine kindergynäkologische Untersuchung durchgeführt werden, ggf. mit entsprechender Beweissicherung.

Die Diagnostik einer Kindesmisshandlung erfolgt multiprofessionell. Psychosoziale Aspekte sollten berücksichtigt und durch den Psychosozialdienst eruiert werden. Dazu zählen auch Gespräche mit dem betroffenen Kind bzw. dem Jugendlichen, dessen Schweigen möglicherweise erst durch eine Vertrauensbasis und der Vermittlung des Gefühls der Sicherheit gelöst werden kann. Eine Kindeswohlgefährdung kann auch durch eine Vernachlässigung entstehen, was zu einem breiten Spektrum an Symptomen führen kann. Deswegen ist im multiprofessionellen Team auch darauf zu achten, dass bei entsprechendem Verdacht eine Ernährungsberaterin (Dystrophie oder Adipositas) ein Mitglied im Kinderschutzteam ist.

\section{Merke \\ Bei Verdacht auf eine Kindesmisshandlung sollte die behandelnde Ärztin bzw. der behandelnde Arzt ein multiprofessionelles Vorgehen einleiten und lokale Strukturen (Kinderschutzgruppe, Kinderschutz- ambulanz) nutzen.}

Nach Sammlung aller Befunde kann innerhalb der Kinderschutzgruppe diskutiert werden, ob die Einleitung weiterer Maßnahmen, z.B. Hinzuziehung des Jugendamts zur Sicherung des Kinderschutzes, notwendig sind. Sollte sich der Verdacht bestätigen, muss ein interdisziplinäres Gespräch mit den Eltern erfolgen. Es ist wichtig, Hilfsangebote für betroffene Familien zu schaffen („Hilfe statt Strafe“), um den Kinderschutz langfristig zu gewährleisten. Die Herausnahme eines Kindes aus einer Familie ist in manchen Fällen erforderlich, stellt aber auch an sich eine belastende Maßnahme für das Kind dar.

Liegt bei dem Kind eine bekannte Erkrankung vor und der Umgang der Eltern bzw. des Kindes (je nach Alter) ist inadäquat, können Maßnahmen, die über die medizinische Versorgung hinausgehen, ebenfalls nur mithilfe des Jugendamtes eingeleitet werden. Ziel ist die nachhaltige Sicherung einer angemessenen medizinischen Versorgung.

\section{KERNAUSSAGEN}

- Es gibt verschiedene Formen von Kindesmisshandlung (körperlich, seelisch, sexuell, Vernachlässigung).

- Bei Frakturen im Kindesalter muss eine Kindesmisshandlung differenzialdiagnostisch in Betracht gezogen werden, insbesondere bei Frakturen im prämobilen Alter.

- Angeborene Skelettdysplasien, Mineralisierungsstörungen oder andere systemische Erkrankungen sollten differenzialdiagnostisch ausgeschlossen werden.

- Durch eine laborchemische Untersuchung kann man zwischen Mineralisierungs-, Synthese- und Resorptionsstörungen unterscheiden.

- Wenn der anamnestisch angegebene Entstehungsmechanismus für die vorliegende Fraktur nicht plausibel erscheint, ist das Röntgen-Skelettscreening das Mittel der Wahl zur Beurteilung des Skelettstatus.

- Der Ausschluss von retinalen Blutungen ist bei Verdacht auf eine Kindesmisshandlung obligat.

- Die ausführliche Anamnese und die körperliche Untersuchung sind umfassend zu dokumentieren. - Dem Verdacht einer Kindesmisshandlung sollte immer multidisziplinär nachgegangen werden.

\section{Interessenkonflikt}

Erklärung zu finanziellen Interessen

Forschungsförderung erhalten: nein; Honorar/geldwerten Vorteil für Referententätigkeit erhalten: nein; Bezahlter Berater/interner Schulungsreferent/Gehaltsempfänger: nein; Patent/Geschäftsanteile/Aktien (Autor/Partner, Ehepartner, Kinder) an Firma: nein. Patent/Geschäftsanteile/Aktien (Autor/Partner, Ehepartner, Kinder) an Firma (Nicht-Sponsor der Veranstaltung): nein.

Erklärung zu nichtfinanziellen Interessen

Die Autorinnen/Autoren geben an, dass kein Interessenkonflikt besteht.

\section{Danksagung}

Besonderer Dank geht an alle Mitglieder der Kinderschutz gruppe der Uniklinik Köln. Teile dieser Publikation wurden durch die „Stiftung Osteogenesis imperfecta Baden-Württemberg" ermöglicht. 


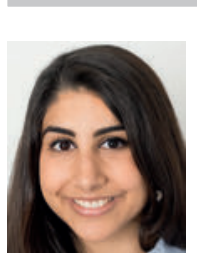

\section{Ramona Ajiri}

Dr. med., 2010-2017 Medizinstudium in Köln. Assistenzärztin, Weiterbildung im Fachgebiet Kinder- und Jugendmedizin an der Universitätsklinik zu Köln. Mitglied der Kinderschutzgruppe der Uniklinik Köln.

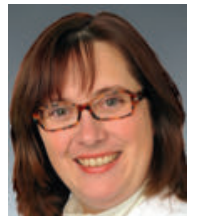

\section{Sibylle Banaschak}

PD Dr. med., 1987-1993 Medizinstudium in Bochum. Facharztausbildung zur Fachärztin für Rechtsmedizin in Lippstadt (Psychiatrie), Bochum (Pathologie) und Münster und Bonn (jeweils Rechtsmedizin). Seit 2001 tätig als Oberärztin zunächst in Jena, seit 2006 Leitende Oberärztin am Institut für Rechtsmedizin der Uniklinik Köln. Seit April 2019 Leiterin des Kompetenzzentrums Kinderschutz im Gesundheitswesen NRW.

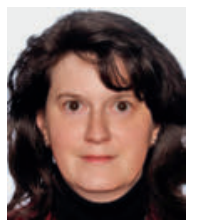

\section{Friederike Körber}

PD Dr. med. 1983-1990 Medizinstudium in Aachen. Facharztausbildung in der Radiologie an der RWTH Aachen. Weiterbildung für den Schwerpunkt Kinderradiologie an der JustusLiebig-Universität Gießen. 2000-2006 Oberärztin im Funktionsbereich Kinderradiologie. Seit 2007 Leitung Funktionsbereich Kinderradiologie des Instituts für diagnostische und interventionelle Radiologie der Universität zu Köln, seit 2017 Schwerpunkt für Kinderradiologie.

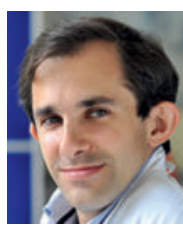

\section{Oliver Semler}

PD Dr. med., 1994-2001 Medizinstudium an den Universitäten Köln und Freiburg. Facharztausbildung in Homburg/Saar und Köln. Seit 2016 Leiter des Zentrums für „Seltene Skeletterkrankungen im Kindes- und Jugendalter " an der Uniklinik Köln. Pädiater mit der Zusatzbezeichnung „Pädiatrische Rheumatologie“ und nach DVO zertifizierter Osteologe. Schwerpunkte: Betreuung von Patienten mit seltenen Skeletterkrankungen. Leiter der Kinderschutzgruppe der Uniklinik Köln.

Korrespondenzadresse

PD Dr. Oliver Semler

Klinik für Kinder- und Jugendmedizin

Universität Köln

Kerpener Straße 62

D-50931 Köln

joerg.semler@uk-koeln.de

\section{Wissenschaftlich verantwortlich}

\section{gemäß Zertifizierungsbestimmungen}

Wissenschaftlich verantwortlich gemäß Zertifizierungsbestimmungen für diesen Beitrag ist PD Dr. med. Oliver Semler, Köln.

\section{Literatur}

[1] Sethi D, Bellis M, Hughes K et al. European report on preventing child maltreatment. 2018. Im Internet: www.eurowho.int/ __data/assets/pdf_file/0019/217018/European-Report-onPreventing-Child-Maltreatment.pdf; Stand: 15.08.2019

[2] Mendelson KL. Critical review of "temporary brittle bone disease”. Pediatr Radiol 2005; 35 (10): 1036-1040

[3] Schoenau E, Frost HM. The "muscle-bone unit" in children and adolescents. Calcif Tissue Int 2002; 70 (5): 405-407

[4] Wolff J. Das Gesetz von der Transformation der Knochen. Berlin: Hirschwald Julius Wolff Institut; 1892

[5] Schoenau E, Neu CM, Rauch F, Manz F. Gender-specific pubertal changes in volumetric cortical bone mineral density at the proximal radius. Bone 2002; 31 (1): 110-113

[6] Schoenau E. The "functional muscle-bone unit": a two-step diagnostic algorithm in pediatric bone disease. Pediatr Nephrol 2005; 20 (3): 356-359

[7] Fadell M, Miller A, Trefan L et al. Radiological features of healing in newborn clavicular fractures. Eur Radiol 2017; 27 (5): 2180-2187

[8] Mathew MO, Ramamohan N, Bennet GC. Importance of bruising associated with paediatric fractures: prospective observational study. BMJ 1998; 317 (7166): 1117-1118

[9] AWMF. Kindesmisshandlung, -missbrauch, -vernachlässigung unter Einbindung der Jugendhilfe und Pädagogik (Kinderschutzleitlinie). AWMF-Register-Nr027-069 2019. Im Internet: https://www.awmf.org/leitlinien/aktuelle-leitlinien.html; Stand: 15.08 .2019

[10] Stöver B. Kindesmisshandlung - Bildgebende Diagnostik. Radiologie up2date 2001; 1 (03): 259-274

[11] Stenzel M, Mentzel HJ. Kindesmisshandlung: Radiologische Diagnostik skelettaler Verletzungsfolgen. Radiologie up2date 2012; 12 (02): 103-122

[12] Kleinman PK, Marks SC, Blackbourne B. The metaphyseal lesion in abused infants: a radiologic-histopathologic study. AJR Am J Roentgenol 1986; 146 (5): 895-905

[13] Swischuk LE. Metaphyseal corner fractures in infants: A review. Emerg Radiol 1998; 5 (2): 103-107

[14] Perez-Rossello JM, Connolly SA, Newton AW et al. Whole-body MRI in suspected infant abuse. AJR Am J Roentgenol 2010; 195 (3): 744-750

[15] Zierk J, Arzideh F, Haeckel R et al. Pediatric reference intervals for alkaline phosphatase. Clin Chem Lab Med 2017; 55 (1): 102-110

[16] Hoyer-Kuhn H, Schönau E, Semler O. Knochengesundheit be Kindern. pädiatrie: Kinder- und Jugendmedizin hautnah 2016; 28 (1): $22-27$

[17] Bianchi ML, Baim S, Bishop NJ et al. Official positions of the International Society for Clinical Densitometry (ISCD) on DXA evaluation in children and adolescents. Pediatr Nephrol 2010; 25 (1): $37-47$

[18] Schoenau E, Neu CM, Beck B et al. Bone mineral content per muscle cross-sectional area as an index of the functional muscle-bone unit. J Bone Miner Res 2002; 17 (6): 1095-1101

[19] Stark C, Hoyer-Kuhn H, Knoop K et al. [Secondary forms of osteoporosis. Special features of diagnostics in childhood and adolescence]. Z Rheumatol 2014; 73 (4): 335-341

[20] Semler O, Hoyer-Kuhn H, Netzer C. Osteogenesis imperfecta. Medizinische Genetik 2012; 4: 297-309 
[21] Deutsche Akademie für Kinder- und Jugendmedizin e.V. (DAKJ) und Deutsche Gesellschaft für Kinderschutz in der Medizin (DGKiM). Vorgehen bei Kindesmisshandlung und -vernachlässigung Empfehlungen für Kinderschutz an Kliniken. 2016. Im Internet: www.kindesmisshandlung.de/mediapool/ 32/328527/data/DGKiM-DAKJ_KSG-Leitfaden_1.6123.12.2016.pdf; Stand: 15.08 .2019
Bibliografie

DOI https://doi.org/10.1055/a-0892-5480

Pädiatrie up2date 2019; 14: 311-327

(c) Georg Thieme Verlag KG Stuttgart · New York ISSN 1611-6445 


\section{Punkte sammeln auf CME. thiemede}

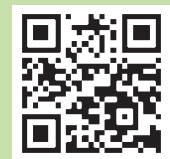

Diese Fortbildungseinheit ist in der Regel 12 Monate online für die Teilnahme verfügbar.

Den genauen Einsendeschluss finden Sie unter https://cme.thieme.de.

Sollten Sie Fragen zur Online-Teilnahme haben, finden Sie unter https://cme.thieme.de/hilfe

eine ausführliche Anleitung. Wir wünschen viel Erfolg beim Beantworten

der Fragen!

Unter https://eref.thieme.de/CXCY528 oder über den QR-Code kommen Sie direkt zur Startseite des Wissenstests.

VNR 2760512019156645630

\section{Frage 1}

Was muss bei Verdacht auf eine misshandlungsbedingte Fraktur differenzialdiagnostisch nicht in Betracht gezogen werden?
A eine angeborene Skelettdysplasie
B ein unbeobachtetes Trauma
C Ullrich-Turner-Syndrom bzw. Trisomie 21
D extreme Mangelernährung mit Nährstoffmangel
E Muskelerkrankungen

\section{Frage 2}

Wie sollte die behandelnde Ärztin bzw. der behandelnde Arzt bei Verdacht auf eine Kindesmisshandlung nicht vorgehen?

A Die Herangehensweise sollte multidisziplinär erfolgen.

B Das Kind sollte beim V.a. Misshandlung stationär aufgenommen werden, wenn die Gefährdung des Kindeswohls nicht ausgeschlossen ist.

C Der Verdacht auf eine Kindesmisshandlung muss den Eltern möglichst frühzeitig kommuniziert werden, um eine juristische Absicherung zu erzielen.

D Eine mögliche Retraumatisierung sollte während der körperlichen Untersuchung vermieden werden.

E Bei der körperlichen Untersuchung sollte neben der Testung der peripheren Durchblutung, die Motorik und Sensibilität untersucht und auch eine Inspektion des Genitalbereichs vorgenommen werden.

\section{Frage 3}

In welchem der folgenden Fälle sollte man am ehesten differenzialdiagnostisch an eine Kindeswohlgefährdung denken?

A 4-jähriges Mädchen, das von einem 2 Meter hohen Klettergerüst gefallen sei und sich eine lineare Schädelfraktur zugezogen hat

B 7 Tage alter männlicher Säugling, $39+2$ SSW, GG $3400 \mathrm{~g}$, Spontanpartus mit einer sonografisch nachgewiesener Klavikulafraktur

C 3-jähriger Junge, der die Treppenstufen der Wohnung heruntergefallen sei und sich eine bilaterale Schädelfraktur zugezogen hat

D 5-jähriger Junge, der unbeobachtet vom Fahrrad gestürzt und auf sein gebeugtes Ellenbogengelenk gefallen sei und eine suprakondyläre Humerusfraktur hat

E 11-jähriger Junge, der von einem Auto angefahren worden sei und eine Femurfraktur hat

\section{Frage 4}

Welcher Befund ist am wenigsten verdächtig auf eine Kindesmisshandlung?

A beidseits Rippenserienfrakturen

B metaphysäre Kantenabsprengungen

C unterschiedlich alte Frakturen an einer Extremität

D eine distale Radiuswulstfraktur

E mehrere Schädelfrakturen

\section{Frage 5}

Was ist nicht mehr Teil des primären Röntgen-Skelettscreenings?
A Fuß a.-p.
B Wirbelsäule seitlich
C Unterarm a.-p.
D Röntgenthorax a.-p.
E Oberschenkel a.-p.

\section{- Weitere Fragen auf der folgenden Seite...}




\section{Frage 6}

Welche 5 Laborparameter sind zur differenzialdiagnostischen Abklärung einer systemischen Knochenerkrankung zunächst notwendig?

A Vitamin D, Phosphat, alkalische Phosphatase, Kalium, Kalzium

B Kalzium, Phosphat, Vitamin D, Parathormon, Blutbild

C Vitamin D, Parathormon, TSH, alkalische Phosphatase, Kalzium

D Kalzium, Phosphat, alkalische Phosphatase, Parathormon, Vitamin D

E Alkalische Phosphatase, Vitamin D, Kalzium, Growth Hormone, Parathormon

\section{Frage 7}

Welche Aussage zur Knochendichtemessung trifft zu?

A Da mit Ultraschall reliable Beurteilungen der Knochenstruktur möglich sind, ist es im Kindesalter nicht erforderlich, ionisierende Verfahren einzusetzen.

B Eine PQCT-Messung ermöglicht die Beurteilung von Wirbelkörpern.

C Zur Abklärung von multiplen Frakturen ist eine GanzkörperMRT-Untersuchung empfohlen, um die Strahlenbelastung durch ein konventionelles Röntgenscreening zu vermeiden.

D Zur Beurteilung der Muskulatur kann die DXA-Technik nicht verwendet werden.

E Die Körpergröße muss bei der Analyse von DXA-Messungen berücksichtigt werden, da sonst große Kinder eine „falsch hohe" Knochenflächendichte haben.

\section{Frage 8}

Welche Aussage zur Molekulargenetik ist richtig?

A Da molekulargenetische Untersuchungen teuer sind, müssen sie bei V.a. Kindesmisshandlung gerichtlich angeordnet werden.

B Da molekulargenetische Untersuchungen eine Kindesmisshandlung nicht beweisen können, sollten sie bei dieser Fragestellung nicht durchgeführt werden.

C Durch den Abgleich der Patienten-DNA mit einem Elternteil können auch unklare Varianten bei dem Patienten in Bezug auf ihre Pathogenität abgeklärt werden.

D Eine umfassende Information des Humangenetikers über die klinische Symptomatik verbessert die Aussage der molekulargenetischen Untersuchung.

E Bei gezieltem Verdacht auf eine Skeletterkrankung sollte möglichst nur ein Einzelgen untersucht werden, da sonst das Risiko für Zufallsbefunde mit unklarer Pathogenität zu stark steigt.

\section{Frage 9}

Was trifft für eine Untersuchung eines Kindes mit dem V.a. eine Fraktur und einem V.a. eine Kindesmisshandlung nicht zu?

A Bei der Erhebung der Anamnese müssen immer ein Facharzt und eine weitere Person als Zeuge anwesend sein.

B Es sollte eine vertrauensvolle Umgebung für die Befragung und die Untersuchung geschaffen werden.

C Radiologische Untersuchungen sollten berücksichtigen, dass Schmerzlokalisationen von Kindern manchmal ungenau sind.

D Bei einer unklaren Situation sollte das Kind immer stationär aufgenommen werden.

E Die Versorgung einer Fraktur und das Kindeswohl haben immer Vorrang vor juristischen Aspekten.

\section{Frage 10}

Was sollte bei der Vorgehensweise bei einem V.a. Kindesmisshandlung beachtet werden?

A Das Kind sollte nur in Anwesenheit der Eltern befragt werden, um eine Verängstigung zu vermeiden.

B Suggestivfragen sind vorzuziehen, damit das Kind sich möglichst an den nicht akzidentellen Unfallhergang erinnert und die Anamneseerhebung erleichtert.

C Da die Eltern das Recht auf einen Einblick in die Krankenakte haben, sollte auf eine Dokumentation möglichst verzichtet werden.

D Aufgrund der ärztlichen Schweigepflicht darf der Verdacht auf eine Kindeswohlgefährdung nicht an öffentliche Ämter getragen werden.

E Es sollte eine genaue, juristisch verwertbare, klar leserliche Dokumentation erfolgen. 